\title{
Input-Output Transformation Using the Feedback of Nonlinear Electrical Circuits: Algorithms and Linearization Examples
}

\author{
Sebastian Różowicz $\mathbb{D}$ and Andrzej Zawadzki \\ Faculty of Electrical Engineering, Automatics and Computer Science, Kielce University of Technology, \\ 25-314 Kielce, al. Tysiąclecia Państwa Polskiego 7, Poland \\ Correspondence should be addressed to Sebastian Różowicz; s.rozowicz@tu.kielce.pl
}

Received 26 April 2018; Revised 8 September 2018; Accepted 28 October 2018; Published 6 November 2018

Academic Editor: Vyacheslav Kalashnikov

Copyright (c) 2018 Sebastian Różowicz and Andrzej Zawadzki. This is an open access article distributed under the Creative Commons Attribution License, which permits unrestricted use, distribution, and reproduction in any medium, provided the original work is properly cited.

\begin{abstract}
This paper addresses the problem of nonlinear electrical circuit input-output linearization. The transformation algorithms for linearization of nonlinear system through changing coordinates (local diffeomorphism) with the use of closed feedback loop together with the conditions necessary for linearization are presented. The linearization stages and the results of numerical simulations are discussed.
\end{abstract}

\section{Introduction}

While analyzing actual physical systems we often have to deal with nonlinear problems. Most of them can be described by a system of differential equations in the normal form of Cauchy system. This applies to the dynamics of electrical circuits with concentrated parameters, as well as mechanical or electromechanical systems. The research of such systems is reduced to mathematical analysis of the model, which in a general case is nonlinear. The theory of linear systems of differential equations in the normal form of Cauchy system is explicit with respect to the time derivatives. Therefore, the linearized-preferably linear models of nonlinear systems are sought. This allows considering the dynamic behaviour of the object as a linear one and then going back to the original system by the inverse transformation. This approach may be useful for qualitative research including issues of stability, control, and the existence of periodic solutions.

In general, the nonlinear model describing the dynamics in the state space can be represented by

$$
\begin{aligned}
& \dot{\mathbf{x}}=\mathbf{f}(\mathbf{x})+\sum_{i=1}^{m} \mathbf{g}_{i}(\mathbf{x}) \mathbf{u}_{i}, \quad \mathbf{x} \in \mathbf{R}^{n}, \mathbf{u} \in \mathbf{R}^{m} \\
& \mathbf{y}=\mathbf{h}(\mathbf{x}), \quad \mathbf{y} \in \mathbf{R}^{p}
\end{aligned}
$$

where $\mathbf{f}$ and $\mathbf{g}_{i}$ are smooth vector fields defined on the manifold $M=\mathbf{R}^{\mathrm{n}}$, called the state space, $\mathbf{h}$ is a smooth mapping defined on the state space $M$ into $p$-dimensional space of outputs, and $\mathbf{R}^{p}, \mathbf{h}: M \longrightarrow \mathbf{R}^{p}$.

The methods of differential geometry allow for the linearization of nonlinear systems (1) to the following linear form [1-3]:

$$
\begin{aligned}
\frac{d \mathbf{z}}{d t} & =\mathbf{A z}+\mathbf{B v} \\
\mathbf{y} & =\mathbf{C} \mathbf{z}
\end{aligned}
$$

where $\mathbf{z}$ and $\mathbf{v}$ are new state and input vectors, $\mathbf{w}$ is an output vector in a linearized system, and $\mathbf{A}, \mathbf{B}$, and $\mathbf{C}$ are matrixes of proper size.

Models (2) are obtained as a result of linearizing transformation, transforming the nonlinear system by changing coordinates in the state space. The change consists in the replacement of the original state variables $\mathbf{x}$ with new variables $\mathbf{z}$ describing the system in a new state space [1-13]. The linearizing transformation can be written as

$$
S(\mathbf{x}): \mathbf{x} \Longrightarrow \mathbf{z}
$$

where $S(\mathbf{x})$ is linearizing transformation and $\mathbf{z}(t)$ a new state vector 
After transformation the state vector is of the form:

$$
\mathbf{z}=S(\mathbf{x})
$$

where $S(\mathbf{x})=\left[S_{1}(\mathbf{x}), S_{2}(\mathbf{x}), \ldots, S_{n}(\mathbf{x})\right]^{\mathrm{T}}$ is a mapping defined on an open set of $\mathbf{R}^{n}$ space with $\mathbf{R}^{n}$ values and the following properties:

(i) $S$ is locally invertible; i.e., there exists the inverse mapping $S^{-1}$ (that can be transformed back to the original components of state vector $\mathbf{x}$ ) such that

$$
\mathbf{x}=S^{-1}(\mathbf{z}) \text { for any } \mathbf{z} \text { in } \operatorname{Im} S
$$

where $\operatorname{Im} S$ is the image in the $S$ transformation;

(ii) $S$ and $S^{-1}$ are smooth mappings.

The transformation of this kind is called a diffeomorphism $[2,3,10,14]$.

The design methods of nonlinear control systems have been developed for many years. Based upon differential geometry the necessary and sufficient conditions for linearization of nonlinear control system (by changing coordinates) have been obtained [1-4, 14-17]. Those conditions, except for the planar case, turn out to be restrictive. Consequently, the natural problem of finding normal forms for nonlinearizable systems appears. The problem is, however, very complex and has been extensively studied.

The systems that cannot be linearized and transform only to quasilinear systems can be further linearized by feedback. In this case, the combination of linearization by the transformation of state variables and input transformation $\mathbf{u}(t)$ with feedback [18-27] should be used. As a result of coordinate transformation and the introduction of feedback, the state vector in a new coordinate system can be represented as

$$
\mathbf{z}=S(\mathbf{x})+\text { feedback }
$$

As differential equations are difficult to solve and many systems are not feedback linearizable, the feedback linearization has been extended in various ways (1). One of them is the method based upon the theory of singularities of vector fields and distributions discussed in [16, 28-31]. Other methods were proposed by a few authors including Gardner [32] describing the geometry of feedback equivalence [33$36]$, by Bonnard [37, 38] and by Jakubczyk [39, 40] using basing on the Hamiltonian formalism for optimal control problems. The concepts of partial linearization, approximate linearization, pseudolinearization, extended linearization, etc.; see, e.g., [14, 17, 22, 41-47], introduced. Numerous papers on linearization of nonlinear system with the use of closed feedback loop inspired by Kang and Krener [24] was presented for single-input systems by Tall and Respondek [48] and for multi-input systems by Tall [49]. In [50] the authors solved these two problems by defining algorithms allowing the explicit computation of the linearizing state (resp. feedback) coordinates for any nonlinear control system that is linearizable (resp., feedback linearizable).
Transformation of nonlinear system into linear one by linearization (with the local equivalence of the system dynamics ensured) is very useful in solving practical problems concerning nonlinear behaviour of objects. This approach simplifies the analysis of nonlinear systems. Given the relevance of the problems the attempt has been made to develop efficient algorithms for linearization of a given certain class of nonlinear systems.

This paper aims to develop linearization algorithms of input-output nonlinear systems through the transformation of state space with the use of closed-loop feedback. The use of geometric methods in the theory of electrical circuits has been presented. The linearization of nonlinear SISO systems has been discussed and extended for the linearization of MIMO systems. The elements of Lie algebra $[3,5,6,51,52]$ have been used for the construction of linearizing transformation (6). It is worth mentioning that the linearization techniques have already proved to be very useful and are still of interest nowadays.

The paper is organized as follows. Section 2 provides basic definitions and theorems concerning the conditions to be met for the linearization of a nonlinear system. The elements of the Lie algebra used to construct a new base of state space are presented. The algorithms employed for the linearization of nonlinear mathematical models and the examples (with emphasis on electrical circuits) proving that the algorithms are correct are given in Section 3. Conclusions and comments are presented in Section 4, followed by the bibliography.

\section{Preliminaries}

This section outlines some basic definitions and concepts of differential geometry. The efforts were made to present them in a simplified and concise form. The indepth explanation can be found in the bibliography. In the analysis of nonlinear systems the operation including real function $h$ and vector field $\mathrm{f}$ defined on the space manifold $M$ deserves particular attention. The result of the operation is a smooth real-valued function defined for each $\mathrm{x}$ from the $M$ set as follows.

Definition 1. Let $h$ mapping $\mathbf{R}^{n} \longrightarrow \mathbf{R}^{n}$ be a smooth scalar function of $n$ variables, $\mathbf{x}=\left(x_{1}, x_{2}, \ldots, x_{n}\right)^{\mathrm{T}}, \in \mathbf{R}^{n}$, and $\mathbf{f}$ : $\mathbf{R}^{n} \longrightarrow \mathbf{R}^{n}$ a vector field defined on manifold $M=\mathbf{R}^{n}$; then the Lie derivative of scalar function $h(\mathbf{x})=h\left(x_{1}, x_{2}, \ldots x_{n}\right)$ in the direction of field $\mathbf{f}$ is a scalar function given by the formula:

$$
L_{\mathbf{f}} h=\frac{\partial h}{\partial \mathbf{x}} \circ \mathbf{f}=\sum_{i=1}^{n} \frac{\partial h}{\partial x_{i}} f_{i}
$$

There exists the following recurrence formula for this derivative:

$$
\begin{aligned}
& L_{\mathbf{f}}^{0} h=h \quad \text { - zero derivative for } \quad i=1,2, \ldots \\
& L_{\mathbf{f}}^{n} h=L_{\mathbf{f}}\left(L_{\mathbf{f}}^{n-1} \mathrm{~h}\right) \quad \text { - nth derivative }
\end{aligned}
$$


The second operation concerns vector fields $\mathbf{f}$ and $\mathbf{g}$ defined on the manifold $M=\mathbf{R}^{n}$ space. The result of the operation (defined below) is a new smooth vector field.

Definition 2. Let $\mathbf{f}$ and $\mathbf{g}$ be vector fields defined on the manifold $M=\mathbf{R}^{n}$. Lie brackets of the vector field are called the third vector field, defined by the following relation:

$$
[\mathbf{f}, \mathbf{g}]=\frac{\partial \mathbf{g}}{\partial \mathbf{x}} \circ \mathbf{f}-\frac{\partial \mathbf{f}}{\partial \mathbf{x}} \circ \mathbf{g},
$$

where o- scalar product.

For such notation of square brackets we obtain the recurrence formulae

$$
\begin{aligned}
& a d_{\mathbf{f}}^{0} \mathbf{g}=\mathbf{g} \\
& a d_{\mathbf{f}}^{i} \mathbf{g}=\left[\mathbf{f}, a d_{\mathbf{f}}^{i-1} \mathbf{g}\right]
\end{aligned}
$$

The direct linearization occurs for nonlinear systems for which the vector field represented by Lie brackets $\left\{\mathbf{g}, a d_{\mathbf{f}}^{1} \mathbf{g}, a d_{\mathbf{f}}^{2} \mathbf{g}, \ldots, a d_{\mathbf{f}}^{n-1} \mathbf{g}\right\}$ satisfies the conditions of Theorem 3. After introducing the notations of the conditions of controllability (C) and observability $(\mathrm{O})$ Theorem 3 takes the following form.

Theorem 3. The following conditions are equivalent [53]:

(a) System (1) is equivalent, under a (local) diffeomorphism $\mathbf{z}=S(\mathbf{x})$, to the controllable and observable linear system (2).

(b) System (1) satisfies (C), (O), and $L_{g_{i_{1}}} L_{g_{i_{2}}} \cdots L_{g_{i_{k}}} h_{j}=0$, for all $1 \leq j \leq p$, all $\kappa \geq 1$, and all $0 \leq i_{q} \leq m$ (where $g_{0}=f$ ), when at least two of the $i_{q}$ 's differ from 0 . (c) System (1) satisfies (C), (O), and $L_{g_{j}} L_{f}^{q} h_{i}=$ constant for all $1 \leq j \leq m$, all $1 \leq i \leq p$, and all $0 \leq q \leq \kappa_{j}+\mu_{i}-1$.

(d) System (1) satisfies (C), (O), and $\left[a d_{\mathbf{f}}^{q} \mathbf{g}_{j}, a d_{\mathrm{f}}^{r} \mathbf{g}_{i}\right]=$ 0 , for all $1 \leq i, j \leq m$, all $0 \leq q \leq \kappa_{j}, 0 \leq r \leq K_{i}$ and $L_{g_{i}} L_{f}^{q} h_{i}=$ constant for all $1 \leq j \leq m$, all $1 \leq i \leq p$, and all $0 \leq q \leq \kappa_{j}-1$.

(e) System (1) satisfies (C), (O), and $d L_{f}^{\mu_{i}} h_{i}=$ $\sum_{j=1}^{p} \sum_{k=0}^{\mu_{i}-1} C_{j k}^{i} d L_{f}^{k} h_{j}$, for all $1 \leq i \leq p$, where $C_{j k}^{i}$ s are real numbers and $L_{g_{i}} L_{f}^{q} h_{i}=$ constant for all $1 \leq j \leq m$, all $1 \leq i \leq p$, and all $0 \leq q \leq \mu_{i}-1$.

The proof of Theorem 3 and the extensive discussion of conditions are presented in [53].

In the analysed model of the nonlinear system (1) the output of $y(t)$ is related to input $u(t)$ through the state variables and the nonlinear equation of state. A simple and direct form of the relation between the output $y(t)$ (or its derivatives) and the input $u(t)$ should be found. The starting point to determine diffeomorphism transforming the system (1) into linear system (2) is the definition of a relative degree of the system, sometimes called the characteristic number [13].

Definition 4. A relative degree $r_{1}(\mathbf{x}), \ldots, r_{p}(\mathbf{x})$ of a smooth nonlinear system includes the smallest natural numbers such that for each $j \in\{1, \ldots, p\}$ :

$$
\text { (i) } \quad L_{\mathbf{g}} L_{\mathbf{f}}^{k} h_{j}(\mathbf{x})=\left(L_{g_{1}} L_{\mathbf{f}}^{k} h_{j}(\mathbf{x}), \ldots, L_{g_{m}} L_{\mathbf{f}}^{k} h_{j}(\mathbf{x})\right)=0, \quad k=0, \ldots, r_{j}-2, \forall \mathbf{x} \in U
$$

(ii)

$$
L_{\mathbf{g}} L_{\mathbf{f}}^{r_{j}(\mathbf{x})-1} h_{j}(\mathbf{x})=\left(L_{g_{1}} L_{\mathbf{f}}^{r_{j}(\mathbf{x})-1} h_{j}(\mathbf{x}), \ldots, L_{g_{m}} L_{\mathbf{f}}^{r_{j}(\mathbf{x})-1} h_{j}(\mathbf{x})\right) \neq 0, \quad \text { for certain } \mathbf{x} \in U
$$

If $L_{\mathbf{g}} L_{\mathbf{f}}^{k} h_{j}(\mathbf{x})=\left(L_{g_{1}} L_{\mathbf{f}}^{k} h_{j}(\mathbf{x}), \ldots, L_{g_{m}} L_{\mathbf{f}}^{k} h_{j}(\mathbf{x})\right)=0 \forall \mathrm{k} \geq 0$ and $\mathbf{x} \in U$, it is assumed that $r_{j}=\infty$.

It should be emphasized that each number $r_{i}$ is associated with the $i$-th output of the $h_{i}$ system. It is also worth stressing that by differentiating the $y_{i}$ output with respect to time $r_{i}$ times we obtain

$$
y_{i}^{r_{i}}=L_{\mathbf{f}}^{r_{i}} h_{i}(\mathbf{x})+L_{g_{i}} L_{\mathbf{f}}^{r_{i}} h_{i}(\mathbf{x}) u_{i}, \quad i \in\{1, \ldots, p\}
$$

That is, $r_{i}$ is the number that tells how many times the system output $y_{i}(t)$ should be differentiated to obtain the supply $u_{i}$ in "an explicit form", that is, the linear relation between supply $u_{i}$ and output $y_{i}$.
If the characteristic numbers of the system are finite, then we can define decoupling matrix:

$$
\mathbf{E}(\mathbf{x})=\left[\begin{array}{c}
L_{g_{1}} L_{\mathbf{f}}^{r_{1}-1} h_{1}, \ldots, L_{g_{m}} L_{\mathbf{f}}^{r_{1}-1} h_{1} \\
\vdots \\
L_{g_{1}} L_{\mathbf{f}}^{r_{p}-1} h_{p}, \ldots, L_{g_{m}} L_{\mathbf{f}}^{r_{p}-1} h_{p}
\end{array}\right]
$$

The method of determining the coordinate (local transformation) of a linearized system is as follows.

Lemma 5. Let the system have a relative degree $r_{1}, \ldots, r_{p}$ at the point $\mathbf{x}_{0}$, and let the rank of decoupling matrix $\mathbf{E}\left(\mathbf{x}_{\mathrm{o}}\right)$ be p. Then $r_{1}+\ldots+r_{\mathrm{p}} \leq n$ and for $1 \leq i \leq p$ coordinate transformation is 


$$
\begin{gathered}
S_{1}^{i}(\mathbf{x})=h_{i}(\mathbf{x}) \\
S_{2}^{i}(\mathbf{x})=L_{\mathbf{f}} h_{i}(\mathbf{x}) \\
\vdots \\
S_{r_{i}}^{i}(\mathbf{x})=L_{\mathbf{f}}^{r_{i}-1} h_{i}(\mathbf{x})
\end{gathered}
$$

New coordinates for $1 \leq i \leq p$ are defined as

$$
\begin{aligned}
{\left[z_{1}, z_{2}, \ldots, z_{r_{i}}\right] } & =\left[S_{1}^{i}, S_{2}^{i}, \ldots, S_{r_{i}}^{i}\right] \\
& =\left[h_{1}(\mathbf{x}), L_{\mathbf{f}} h_{1}(\mathbf{x}), \ldots L_{\mathbf{f}}^{r_{i}-1} h_{p}(\mathbf{x})\right]
\end{aligned}
$$

Their derivatives are determined as follows:

$$
\begin{aligned}
\dot{z}_{1} & =\frac{d}{d t}\left(h_{1}(\mathbf{x})\right)=\frac{d h_{1}}{d \mathbf{x}}(\mathbf{x}) \dot{\mathbf{x}}=\frac{d h_{1}}{d \mathbf{x}}(\mathbf{x}) \mathbf{f}(\mathbf{x}) \\
& =L_{\mathbf{f}} h_{1}(\mathbf{x})=z_{2}
\end{aligned}
$$

By calculating other components in the same way we obtain a general form of linearized system of

$$
\begin{gathered}
\dot{z}_{1}^{i}=z_{2}^{i} \\
\dot{z}_{1}^{i}=z_{3}^{i} \\
\vdots \\
\dot{z}_{r_{i}-1}^{i}=z_{r_{i}}^{i} \\
\dot{z}_{r_{i}}^{i}=b_{i}(\mathbf{z}, \mathbf{w})+\sum_{i=1}^{m} a_{i j}(\mathbf{z}, \mathbf{w}) u_{j}(\mathbf{z})
\end{gathered}
$$

The output equation is written as

$$
y_{i}=z_{1}^{i}
$$

The above equations represent a normal form of equations describing a nonlinear system with $m$ inputs and $p$ outputs, with a relative degree $r_{1}, \ldots, r_{p}$ at the point $\mathbf{x}_{\mathrm{o}}$.

It should be noted that in (15a) coefficients $a_{\mathrm{ij}}$ are equal to $\mathbf{E}(\mathbf{x})$ matrix elements, where $\mathbf{x}$ is replaced by $\mathbf{S}^{-1}(\mathbf{z})$, and coefficients $b_{\mathrm{i}}(\mathbf{z}, \mathbf{w})$ are the elements of the vector:

$$
\mathbf{b}(\mathbf{x})=\left[L_{\mathbf{f}}^{r_{1}} h_{1}(\mathbf{x}), L_{\mathbf{f}}^{r_{2}} h_{2}(\mathbf{x}), \cdots, L_{\mathbf{f}}^{r_{p}} h_{p}(\mathbf{x})\right]^{\mathrm{T}}
$$

where $\mathbf{x}$ is replaced by $\mathbf{S}^{-1}(\mathbf{z})$.

Intuitively, it seems that a set of nonlinear systems that can be transformed by coordinate change is very small. Therefore, if a nonlinear system does not meet the conditions of Theorem 3 we consider the transformation of the original model into an equivalent one with simpler dynamics resulting from the transformation of the system state and feedback (6).

Definition 6. Regular state static feedback of nonlinear system (1) is defined as

$$
\mathbf{u}=\alpha(\mathbf{x})+\beta(\mathbf{x}) \mathbf{v}
$$

where $\mathbf{u}=\left(u_{1}, \ldots, u_{\mathrm{m}}\right)^{\mathrm{T}}$ and $\alpha: M=\mathbf{R}^{m}$ and $\beta: M=\mathbf{R}^{m \times m}$ are smooth mappings with the property that matrix $\beta(\mathbf{x})$ is nonsingular for any $\mathbf{x}$ and $\mathbf{v}=\left(v_{1}, \ldots, v_{\mathrm{m}}\right)$ is a new supply vector.

The application of regular, static feedback to the system (1) results in

$$
\begin{aligned}
\frac{d \mathbf{x}(t)}{d t}= & \mathbf{f}(\mathbf{x}(t))+\sum_{i=1}^{m} \mathbf{g}_{i}(\mathbf{x}) \alpha_{i}(\mathbf{x}) \\
& +\sum_{j=1}^{m}\left(\sum_{i=1}^{m} \mathbf{g}_{i}(\mathbf{x}) \beta_{j i}(\mathbf{x})\right) v_{j}
\end{aligned}
$$

which is of the same form as the considered nonlinear system but now with the newly defined inputs (supplies) $\left(v_{1}, \ldots, v_{\mathrm{m}}\right)$.

Regular, static feedback can be used to solve a much larger group of linearization than is the case of transformation of state coordinates. This allows for the linearization of the system, its decoupling, and decomposition. On the other hand, the use of feedback alters the dynamics of the original system.

Let us consider the case of a nonlinear system (1) having $m$ inputs and $m$ outputs described by

$$
\begin{aligned}
& \dot{\mathbf{x}}=\mathbf{f}(\mathbf{x})+\sum_{i=1}^{m} \mathbf{g}_{i}(\mathbf{x}) \mathbf{u}_{i} \\
& \mathbf{y}=\mathbf{h}(\mathbf{x})=\left[h_{1}(\mathbf{x}), \ldots, h_{m}(\mathbf{x})\right]
\end{aligned}
$$

Let the outputs of the system be divided into $m$ disjoint blocks.

Here, the task of linearization consists in finding a new supply of the system (19) so that each of the $m$ output blocks depends on one supply only; i.e., its outputs are decoupled from the inputs.

Definition 7. Nonlinear system (19) has inputs decoupled from outputs around the point $\mathbf{x}_{\mathrm{o}}$ if there exists a neighbourhood $V$ of $\mathbf{x}_{\mathrm{o}}$ point in which $L_{\mathbf{g}_{j}} L_{\tau_{1}} \ldots L_{\tau_{k}} h(\mathbf{x})=0$ for each $\mathbf{x} \in V$ for any vector field $\boldsymbol{\tau}_{1}, \boldsymbol{\tau}_{2}, \ldots, \boldsymbol{\tau}_{k}$, from the set $\left.\left\{\mathbf{f}, g_{1}, \ldots, g_{\mathrm{m}}\right\}\right)$ and if a relative degree of the system $r_{1}, \ldots r_{m}$ is finite and constant on $V$.

In order to solve the problem of linearization for the system with multiple outputs and multiple inputs with the initial state $\mathbf{x}_{\mathrm{o}}$ one should find a regular, static feedback defined in the neighbourhood $V$ of the $\mathbf{x}_{\mathrm{o}}$ point such that each output $y_{\mathrm{i}}$ is affected by one input $v_{\mathrm{i}}, 1 \leq i \leq m$. The feedback in this case is

$$
\left[\begin{array}{c}
u_{1} \\
u_{2} \\
\vdots \\
u_{m}
\end{array}\right]=\mathbf{E}^{-1}(\mathbf{x})\left[\left(\begin{array}{c}
v_{1} \\
v_{2} \\
\vdots \\
v_{m}
\end{array}\right)-\left(\begin{array}{c}
b_{1}(\mathbf{x}) \\
b_{2}(\mathbf{x}) \\
\vdots \\
b_{m}(\mathbf{x})
\end{array}\right)\right]
$$

The decoupling matrix $\mathbf{E}(\mathbf{x})$ and the vector $\mathbf{b}(\mathbf{x})$ are given by relations (11) and (16). 


\section{Input-Output Linearization Algorithms of Nonlinear System}

The analysis of nonlinear systems, modelling the inputoutput and undergoing transformations linearizing the system locally includes the following tasks:

(i) linearization of systems with single input and single output-SISO;

(ii) linearization of systems with multiple input and multiple output-MIMO.

The proposals of their algorithmic solutions are presented in the subsequent subsections.

3.1. Algorithm Linearizing Single Input and Single Output (SISO). In most cases the transformation of nonlinear systems does not make them equivalent to linear ones. This is true for the systems that do not satisfy the conditions of Theorem 3. Here, the combination of linearization by transformation of state variables and the transformation of input function $u(t)$ with the use of feedback (17) should be applied.

The following algorithm describes linearization of a nonlinear system with one power supply and one output.

Step 1. We determine a relative degree of the system from Definition 4:

If

$$
\begin{aligned}
L_{\mathbf{g}} L_{\mathbf{f}}^{i} h(\mathbf{x})=0 \quad \text { for } 0 \leq i \leq r-1 \\
L_{\mathbf{g}} L_{\mathbf{f}}^{r-1} h(\mathbf{x}) \neq 0
\end{aligned}
$$

we can define the input transformation of nonlinear system, which means that there exists a regular and static feedback and the system can be linearized.

If, however, $L_{\mathbf{g}} L_{\mathbf{f}}^{k} h(\mathbf{x})=0$ for $k \geq 0$ and any $x \in \mathbf{R}^{n}$ the relative degree of the system $r=\infty$ then a regular and static feedback linearizing the system does not exist.

Note 8. The definition of a relative degree of the system can be understood as follows.

By differentiating the output $y$ we obtain

$$
\begin{aligned}
\dot{y} & =\frac{\partial h(\mathbf{x})}{\partial \mathbf{x}} \dot{\mathbf{x}}=\frac{\partial h(\mathbf{x})}{\partial \mathbf{x}}(\mathbf{f}(\mathbf{x})+\mathbf{g}(\mathbf{x}) u) \\
& =L_{\mathbf{f}} h(\mathbf{x})+L_{\mathbf{g}}(\mathbf{x}) u
\end{aligned}
$$

where

$$
\begin{array}{r}
L_{\mathbf{f}} h(\mathbf{x})=\frac{\partial h(\mathbf{x})}{\partial \mathbf{x}} \mathbf{f}(\mathbf{x}), \\
\text { and } L_{\mathbf{g}} h(\mathbf{x})=\frac{\partial h(\mathbf{x})}{\partial \mathbf{x}} \mathbf{g}(\mathbf{x}) .
\end{array}
$$

Therefore, if $L_{\mathbf{g}} h(\mathbf{x}) \neq 0$ for a given point $x_{\mathrm{o}} \in \Omega_{x}$ then in the neighbourhood of this point the following transformation of system input can be defined:

$$
u=\frac{1}{L_{\mathbf{g}} h(\mathbf{x})}\left(v-L_{\mathbf{f}} h(\mathbf{x})\right)
$$

It is clear that the relation between a new input $v$ and output $y$ is linear.

If, however, $L_{\mathbf{g}} h(\mathbf{x})=0$ for any point $x_{\mathrm{o}} \in \Omega_{x}$ then the differentiation of output should be repeated. Hence

$$
\begin{aligned}
\ddot{y} & =\frac{\partial\left(L_{\mathbf{f}} \mathbf{h}(\mathbf{x})\right)}{\partial \mathbf{x}} \dot{x}=\frac{\partial\left(L_{\mathbf{f}} \mathbf{h}(\mathbf{x})\right)}{\partial \mathbf{x}}(\mathbf{f}(\mathbf{x})+\mathbf{g}(\mathbf{x}) u) \\
& =L_{\mathbf{f}}^{2} \mathbf{h}(\mathbf{x})+L_{\mathbf{g}} L_{\mathbf{f}} \mathbf{h}(\mathbf{x}) u
\end{aligned}
$$

And again if $L_{\mathbf{g}} L_{\mathbf{f}} h(\mathbf{x}) \neq 0$ then a new supply can be written as

$$
u=\frac{1}{L_{\mathbf{g}} L_{\mathbf{f}} h(\mathbf{x})}\left(v-L_{\mathbf{f}}^{2} h(\mathbf{x})\right)
$$

Otherwise, the differentiation procedure should be continued up to the $i^{\text {th }}$ step where for some natural $r$ we obtain $L_{\mathbf{g}} L_{\mathbf{f}}^{r} h(\mathbf{x}) \neq 0$ for some $x_{\mathrm{o}} \in \Omega_{x}$.

The output differentiation is expressed by the following general relation:

$$
\begin{aligned}
y^{(i)} & =\frac{\partial\left(L_{\mathbf{f}}^{i-1} h(\mathbf{x})\right)}{\partial \mathbf{x}} \dot{x}=\frac{\partial\left(L_{\mathbf{f}}^{i-1} h(\mathbf{x})\right)}{\partial \mathbf{x}}(\mathbf{f}(\mathbf{x})+\mathbf{g}(\mathbf{x}) u) \\
& =L_{\mathbf{f}}^{i} h(\mathbf{x})+L_{\mathbf{g}} L_{\mathbf{f}}^{i-1} h(\mathbf{x}) u
\end{aligned}
$$

Finally, a new supply is

$$
u=\frac{1}{L_{\mathbf{g}} L_{\mathbf{f}}^{r-1} h(\mathbf{x})}\left(v-L_{\mathbf{f}}^{r} h(\mathbf{x})\right)
$$

which leads to the relation:

$$
y^{(r)}=v
$$

and we obtain the linear relation between input $v$ and output $y$.

Step 2. If a relative degree of the system $r=n$ then linearizing transformation $S(\mathbf{x})$ is determined by relation (12). Hence, for the system with single input and single output we get

$$
\begin{gathered}
S_{1}(\mathbf{x})=h(\mathbf{x}) \\
S_{2}(\mathbf{x})=L_{\mathbf{f}} h(\mathbf{x}) \\
\vdots \\
S_{r}(\mathbf{x})=L_{\mathbf{f}}^{r-1} h(\mathbf{x})
\end{gathered}
$$

Step 3. We determine the state vector in new coordinates $\mathbf{z}=$ $S(\mathbf{x})=\left[S_{1}(\mathbf{x}), S_{2}(\mathbf{x}), \ldots, S_{r}(\mathbf{x}),\right]^{\mathrm{T}}$.

Step 4. We determine a regular, static feedback linearizing the analyzed system using (17) and new input (supply) $v$. 
Step 5. For one-dimensional case the system dynamics in the new coordinates and with a new supply $v$ is obtained as follows:

$$
\begin{gathered}
\dot{z}_{1}=\frac{\partial S_{1}}{\partial \mathbf{x}} \frac{d \mathbf{x}}{d t}=\frac{\partial h}{\partial \mathbf{x}} \frac{d \mathbf{x}}{d t} \\
\dot{z}_{2}=\frac{\partial S_{2}}{\partial \mathbf{x}} \frac{d \mathbf{x}}{d t}=\frac{\partial\left(L_{\mathbf{f}} h\right)}{\partial \mathbf{x}} \frac{d \mathbf{x}}{d t} \\
\vdots \\
\dot{z}_{r-1}=\frac{\partial S_{r-1}}{\partial \mathbf{x}} \frac{d \mathbf{x}}{d t}=\frac{\partial\left(L_{\mathbf{f}}^{r-2} h\right)}{\partial \mathbf{x}} \frac{d \mathbf{x}}{d t} \\
\dot{z}_{r}=v
\end{gathered}
$$

Hence, we get the equations of states (15a) and (15b):

$$
\begin{gathered}
\dot{z}_{1}=S_{2}(x(t))=L_{\mathbf{f}} h(x(t))=z_{2}(t) \\
\dot{z}_{2}=S_{3}(x(t))=L_{\mathbf{f}}^{2} h(x(t))=z_{3}(t) \\
\vdots \\
\dot{z}_{r-1}=S_{r}(x(t))=L_{\mathbf{f}}^{r-1} h(x(t))=z_{r}(t) \\
\dot{z}_{r}=v
\end{gathered}
$$

and output

$$
y=z_{1}
$$

Step 6. If the relative degree of the system $r<n$, then such $n-r$ coordinates $S_{r+1}(\mathbf{x}), \ldots, S_{n}(\mathbf{x})$ are defined as follows: the Jacobian of the mapping (14) is full-rank at a point $x_{\mathrm{o}}$ (with an additional assumption that $\left.L_{\mathbf{g}} S_{i}(\mathbf{x})=0\right)$.

Thus we obtain additional equations of system dynamics:

$$
\begin{aligned}
\dot{z}_{i} & =\frac{\partial S_{i}}{\partial \mathbf{x}}(\mathbf{f}(x(t))+\mathbf{g}(x(t)) u(t)) \\
& =L_{\mathbf{f}} S_{i}(x(t)) u(t)+L_{\mathbf{g}} S(x(t)) u(t)=L_{\mathbf{f}} S_{i}(x(t))
\end{aligned}
$$

Assuming that

$$
q_{i}(\mathbf{z})=L_{\mathbf{f}} S_{i}\left(S^{-1}(\mathbf{z})\right) \quad r+1 \leq i \leq n
$$

the equations are

$$
\dot{z}_{i}=q_{i}(z(t))
$$

Step 7. We determine the state vector in new coordinates:

$$
\mathbf{z}=S(\mathbf{x})=\left[S_{1}(\mathbf{x}), S_{2}(\mathbf{x}), \ldots, S_{r+1}(\mathbf{x}), \ldots, S_{n}(\mathbf{x})\right]^{\mathrm{T}}
$$

Step 8. We determine the scalar function $L_{\mathbf{f}}^{r-1} h(\mathbf{x})$ and define regular feedback by the relation:

$$
u=\frac{1}{L_{\mathbf{g}} L_{\mathbf{f}}^{r-1} h(\mathbf{x})}\left(v-L_{\mathbf{f}}^{r} h(\mathbf{x})\right)
$$

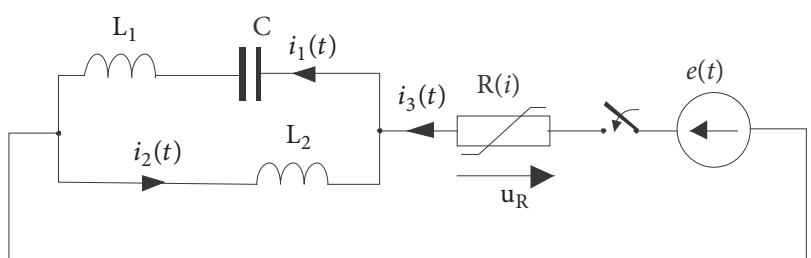

FIGURE 1: Nonlinear circuit of the third order.

Step 9. The system dynamics in new coordinates can be represented by the linearized system of

$$
\begin{gathered}
\dot{z}_{1}=z_{2}, \\
\dot{z}_{2}=z_{3} \\
\vdots \\
\dot{z}_{r-1}=z_{r}, \\
\dot{z}_{r}=v, \\
\dot{z}_{r+1}=q_{r+1} \\
\vdots \\
\dot{z}_{n}=q_{n}
\end{gathered}
$$

and the output

$$
y=z_{1}
$$

Example 9. We consider transient state in the electrical circuit with nonlinear resistor as shown in Figure 1.

Current-voltage characteristics of a nonlinear resistive element are described by a second-degree polynomial:

$$
u(i)=a i^{2}
$$

where $a$ - coefficient of $\left(\Omega A^{-1}\right)$ dimension.

Assuming that the state variables are currents flowing through the inductances and the capacitor voltage, $x_{1}=$ $i_{1}, x_{2}=i_{2}, x_{3}=u_{C}$, the state vector becomes $\mathbf{x}(t)=$ $\left[i_{1}, i_{1}, u_{C}\right]^{\mathrm{T}}=\left[x_{1}(t), x_{2}(t), x_{3}(t)\right]^{\mathrm{T}}$. The state equation modelling the considered electrical circuit is given by

$$
\begin{aligned}
& \dot{x}_{1}=-b \cdot x_{3}-k \cdot\left(x_{1}-x_{2}\right)^{2}+b \cdot e(t) \\
& \dot{x}_{2}=-k \cdot\left(x_{1}-x_{2}\right)^{2}+b \cdot e(t) \\
& \dot{x}_{3}=\frac{1}{C} x_{1}
\end{aligned}
$$


where $b=1 / L_{1}=1 / L_{2}, k=a / L_{1}=a / L_{2}$, and vectors $\mathbf{f}(\mathbf{x}), \mathbf{g}(\mathbf{x}),\left(\mathbf{f}, \mathbf{g} \in \mathbf{R}^{3}, n=3\right)$ are

$$
\begin{aligned}
& \mathbf{f}(\mathbf{x})=\left[\begin{array}{c}
-b x_{3}-k\left(x_{1}-x_{2}\right)^{2} \\
-k\left(x_{1}-x_{2}\right)^{2} \\
\frac{1}{C} x_{1}
\end{array}\right], \\
& \mathbf{g}(\mathbf{x})=\left[\begin{array}{l}
b \\
b \\
0
\end{array}\right]
\end{aligned}
$$

In the circuit described by (41) the output $y(t)$ is related to the power supply $e(t)$ through state variables and nonlinear equation of state. To solve the problem we have to find a new power supply with a regular static feedback. For that purpose we use SISO algorithm for linearization.

Two cases of different output signals (output function $h(\mathbf{x})): y=h(\mathbf{x})=x_{1}-x_{2}$ and $y=h(\mathbf{x})=x_{3}$ are considered.

Case 1. Suppose that we have to find $i_{3}(t)$ current in the branch with a nonlinear resistor.

Since $i_{3}(t)=i_{1}(t)-i_{2}(t)=x_{1}-x_{2}$ the output equation is

$$
y=h(\mathbf{x})=x_{1}-x_{2}
$$

(i) we determine the relative degree of the system.

Directly from the above equation, we can determine the differential of the function $h(\mathbf{x})$ present in the formulas for the Lie derivative of $h$ function along the fields $\mathbf{f}$ and $\mathbf{g}$, respectively:

$$
\frac{\partial h(\mathbf{x})}{\partial(\mathbf{x})}=\left[\begin{array}{lll}
\frac{\partial h(\mathbf{x})}{\partial x_{1}} & \frac{\partial h(\mathbf{x})}{\partial x_{2}} & \frac{\partial h(\mathbf{x})}{\partial x_{3}}
\end{array}\right]=\left[\begin{array}{lll}
1 & -1 & 0
\end{array}\right]
$$

Calculating derivatives one by one

$$
\begin{aligned}
L_{\mathbf{g}} h(\mathbf{x}) & =\frac{\partial h(\mathbf{x})}{\partial \mathbf{x}} \circ \mathbf{g}(\mathbf{x})=\left[\begin{array}{lll}
1 & -1 & 0
\end{array}\right]\left[\begin{array}{lll}
b & b & 0
\end{array}\right]^{\mathrm{T}} \\
& =0 ; \\
L_{\mathbf{g}} L_{\mathbf{f}} h(\mathbf{x}) & =L_{\mathbf{g}}\left(\frac{\partial h(\mathbf{x})}{\partial \mathbf{x}} \circ \mathbf{f}(\mathbf{x})\right)=0 \\
L_{\mathbf{g}} L_{\mathbf{f}}^{2} h(\mathbf{x}) & =L_{\mathbf{g}} L_{\mathbf{f}}\left(L_{\mathbf{f}} h(\mathbf{x})\right)=L_{\mathbf{g}} L_{\mathbf{f}}\left(-b x_{3}\right) \\
& =L_{\mathbf{g}}\left(-\frac{b}{C} x_{1}\right)=\left[\begin{array}{lll}
-\frac{b}{C} & 0 & 0
\end{array}\right]\left[\begin{array}{lll}
b & b & 0
\end{array}\right] \\
& =-\frac{b^{2}}{C}
\end{aligned}
$$

we obtain $L_{\mathrm{g}} L_{\mathbf{f}}^{2} h(\mathbf{x}) \neq 0$, so for any point $\mathbf{x}=\left(x_{1}, x_{2}, x_{3}\right)$ the relative degree of the system is $r(\mathbf{x})=3$. (ii) the next step is to determine the $S(\mathbf{x})$ transformation of state variables and new coordinates:

$$
\begin{aligned}
& S_{1}(\mathbf{x})=h(\mathbf{x})=x_{1}-x_{2}=z_{1} \\
& S_{2}(\mathbf{x})=L_{\mathbf{f}} h(\mathbf{x})=-b x_{3}=z_{2} \\
& S_{3}(\mathbf{x})=L_{\mathbf{f}}^{2} h(\mathbf{x})=-\frac{b}{C} x_{1}=z_{3}
\end{aligned}
$$

(iii) regular, static feedback linearizing the system is of the form:

$$
\begin{aligned}
e & =\frac{1}{L_{\mathbf{g}} L_{\mathbf{f}}^{2} h(\mathbf{x})}\left(v-L_{\mathbf{f}}^{3} h(\mathbf{x})\right) \\
& =-\frac{C}{b^{2}}\left(v-\left(\frac{b^{2}}{C} \cdot x_{3}+\frac{b}{C} \cdot k \cdot\left(x_{1}-x_{2}\right)^{2}\right)\right)
\end{aligned}
$$

Hence, a new supply is

$$
v=\frac{b^{2}}{C} \cdot x_{3}+\frac{b}{C} \cdot k \cdot\left(x_{1}-x_{2}\right)^{2}-\frac{b^{2}}{C} \cdot e
$$

(iv) we determine normal form of linearized system for the above feedback:

$$
\begin{aligned}
\dot{z}_{1}(\mathbf{x}) & =\frac{d}{d t} S_{1}(\mathbf{x})=\frac{d}{d t} h(\mathbf{x})=\frac{d}{d t}\left(x_{1}-x_{2}\right) \\
& =\dot{x}_{1}-\dot{x}_{2}=-b \cdot x_{3}=z_{2}(\mathbf{x}) \\
\dot{z}_{2}(\mathbf{x}) & =\frac{d}{d t} S_{2}(\mathbf{x})=\frac{d}{d t} L_{\mathbf{f}} h(\mathbf{x})=\frac{d}{d t}\left(-b \cdot x_{3}\right) \\
& =-b \cdot \dot{x}_{3}=-\frac{b}{C} \cdot x_{1}=z_{3}(\mathbf{x}) \\
\dot{z}_{3}(\mathbf{x}) & =\frac{d}{d t} S_{3}(\mathbf{x})=\frac{d}{d t} L_{\mathbf{f}}^{2} h(\mathbf{x})=\frac{d}{d t}\left(-\frac{b}{C} \dot{x}_{1}\right) \\
& =\frac{b^{2}}{C} \cdot x_{3}+\frac{b}{C} k \cdot\left(x_{1}-x_{2}\right)^{2}-\frac{b^{2}}{C} \cdot e=v(\mathbf{x})
\end{aligned}
$$

The system dynamics of new coordinate system can be represented by the matrix:

$$
\begin{aligned}
{\left[\begin{array}{l}
\dot{z}_{1} \\
\dot{z}_{2} \\
\dot{z}_{3}
\end{array}\right] } & =\left[\begin{array}{lll}
0 & 1 & 0 \\
0 & 0 & 1 \\
0 & 0 & 0
\end{array}\right]\left[\begin{array}{l}
z_{1} \\
z_{2} \\
z_{3}
\end{array}\right]+\left[\begin{array}{l}
0 \\
0 \\
1
\end{array}\right] v \\
y & =\left[\begin{array}{l}
1 \\
0 \\
0
\end{array}\right]\left[\begin{array}{l}
z_{1} \\
z_{2} \\
z_{3}
\end{array}\right]
\end{aligned}
$$

The correct work of the algorithm is confirmed by the results obtained by the simulation of linearized system. The results are presented in Figure 2. 


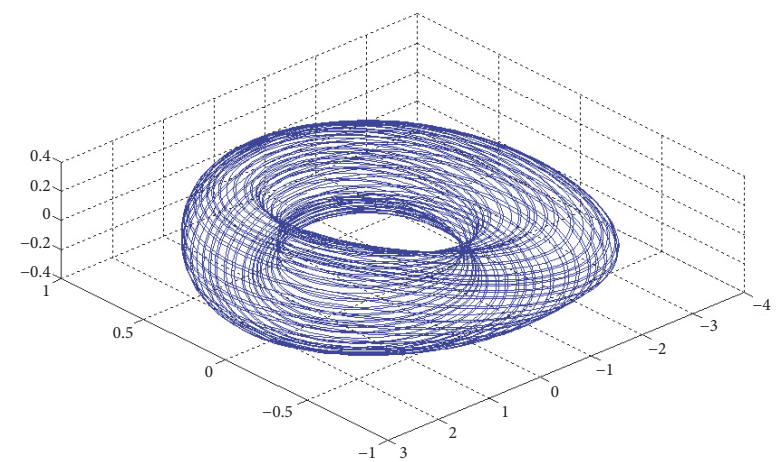

FIGURE 2: Linearized system.

Figure 2 shows the numerical solutions of (48) after transformation of state variables $\mathbf{z}(t)$ of the linear system into variables $\mathbf{x}(t)$ of nonlinear system.

Case 2. Now let us find voltage on the capacitor $u_{c}=x_{3}$. The output function is

$$
y=h(\mathbf{x})=x_{3}
$$

so the differential of the $h(\mathbf{x})$ function is

$$
\frac{\partial h(\mathbf{x})}{\partial(\mathbf{x})}=\left[\begin{array}{lll}
\frac{\partial h(\mathbf{x})}{\partial x_{1}} & \frac{\partial h(\mathbf{x})}{\partial x_{2}} & \frac{\partial h(\mathbf{x})}{\partial x_{3}}
\end{array}\right]=\left[\begin{array}{lll}
0 & 0 & 1
\end{array}\right]
$$

(i) by calculating the relative degree of the system we obtain

$$
\begin{gathered}
L_{\mathbf{g}} h(\mathbf{x})=\frac{\partial h(\mathbf{x})}{\partial \mathbf{x}} \circ \mathbf{g}(\mathbf{x})=\left[\begin{array}{lll}
0 & 0 & 1
\end{array}\right]\left[\begin{array}{lll}
b & b & 0
\end{array}\right]^{\mathrm{T}}=0, \\
L_{\mathbf{g}} L_{\mathbf{f}} h(\mathbf{x})=L_{\mathbf{g}}\left(\frac{\partial h(\mathbf{x})}{\partial \mathbf{x}} \circ \mathbf{f}(\mathbf{x})\right)=L_{\mathbf{g}}\left(\frac{1}{C} x_{1}\right)=\frac{b}{C}
\end{gathered}
$$

that for any point $\mathbf{x}=\left(x_{1}, x_{2}, x_{3}\right)$ the relative degree of the system is $r(\mathbf{x})=2$.

Since $r<n$ using the algorithm in accordance with Step 6 we have to determine the third component of transformation $S(\mathbf{x})$ by solving the partial equation:

$$
\begin{aligned}
& \frac{\partial S_{3}(\mathbf{x})}{\partial \mathbf{x}} \circ \mathbf{g}(\mathbf{x}) \\
& =\left[\begin{array}{lll}
\frac{\partial S_{3}(\mathbf{x})}{\partial x_{1}} & \frac{\partial S_{3}(\mathbf{x})}{\partial x_{2}} & \frac{\partial S_{3}(\mathbf{x})}{\partial x_{3}}
\end{array}\right]\left[\begin{array}{lll}
b & b & 0
\end{array}\right]^{\mathrm{T}} \\
& \quad=b \cdot \frac{\partial S_{3}(\mathbf{x})}{\partial x_{1}}+b \cdot \frac{\partial S_{3}(\mathbf{x})}{\partial x_{2}}
\end{aligned}
$$

The function, which satisfies the above equation and the condition $L_{\mathbf{g}} S_{i}(\mathbf{x})=0$, can be given by the formula:

$$
S_{3}(\mathbf{x})=x_{1}-x_{2}
$$

Thus, the new state variables are of the form:

$$
\begin{aligned}
& z_{1}=S_{1}(\mathbf{x})=h(\mathbf{x})=x_{3}, \\
& z_{2}=S_{2}(\mathbf{x})=L_{\mathbf{f}} h(\mathbf{x})=\frac{1}{C} x_{1}, \\
& z_{3}=S_{3}(\mathbf{x})=x_{1}-x_{2}
\end{aligned}
$$

The feedback is given as

$$
\begin{aligned}
e & =\frac{1}{L_{\mathbf{g}} L_{\mathbf{f}} h(\mathbf{x})}\left(v-L_{\mathbf{f}}^{2} h(\mathbf{x})\right) \\
& =\frac{C}{b}\left(v-\left(-\frac{b}{C} x_{3}-\frac{k}{C} \cdot\left(x_{1}-x_{2}\right)^{2}\right)\right)
\end{aligned}
$$

And a vector of new supply is

$$
v=-\frac{b}{C} x_{3}-\frac{k}{C} \cdot\left(x_{1}-x_{2}\right)^{2}+\frac{b}{C} \cdot e
$$

By determining the normal form of the linearized system we get

$$
\begin{aligned}
\dot{z}_{1}(\mathbf{x}) & =\frac{d}{d t} S_{1}(\mathbf{x})=\frac{d}{d t} h(\mathbf{x})=\frac{d}{d t}\left(x_{3}\right)=\dot{x}_{3}=\frac{1}{C} x_{1} \\
& =z_{2}(\mathbf{x}) \\
\dot{z}_{2}(\mathbf{x}) & =\frac{d}{d t} S_{2}(\mathbf{x})=\frac{d}{d t} L_{\mathbf{f}} h(\mathbf{x})=\frac{d}{d t}\left(\frac{1}{C} x_{1}\right)=\frac{1}{C} \cdot \dot{x}_{1} \\
& =\left(-\frac{b}{C} \cdot x_{3}-\frac{k}{C} \cdot\left(x_{1}-x_{2}\right)^{2}+\frac{b}{C} \cdot e\right)=v \\
\dot{z}_{3}(\mathbf{x}) & =\frac{d}{d t} S_{3}(\mathbf{x})=\frac{d}{d t}\left(x_{1}-x_{2}\right)=\left(\dot{x}_{1}-\dot{x}_{2}\right) \\
& =-b \cdot x_{3}=-b \cdot z_{1}(\mathbf{x})
\end{aligned}
$$

Dynamics of the system in new coordinates is written as

$$
\begin{aligned}
& \dot{z}_{1}=z_{2} \\
& \dot{z}_{2}=v \\
& \dot{z}_{3}=-b \cdot z_{1} \\
& y=z_{1}
\end{aligned}
$$$$
\text { or in matrix notation : }\left[\begin{array}{l}
\dot{z}_{1} \\
\dot{z}_{2} \\
\dot{z}_{3}
\end{array}\right]
$$$$
=\left[\begin{array}{ccc}
0 & 1 & 0 \\
0 & 0 & 0 \\
-b & 0 & 0
\end{array}\right]\left[\begin{array}{l}
z_{1} \\
z_{2} \\
z_{3}
\end{array}\right]+\left[\begin{array}{l}
0 \\
1 \\
0
\end{array}\right] v ;
$$$$
y=\left[\begin{array}{l}
1 \\
0 \\
0
\end{array}\right]\left[\begin{array}{l}
z_{1} \\
z_{2} \\
z_{3}
\end{array}\right]
$$

The resulting system of equations is equivalent to the system described by the mathematical model considered for Case 1. 
3.2. Linearizatrion Algorithm for Multi-Input and Multi Output Systems (MIMO). Let us consider a nonlinear system of $m$ inputs and $m$ outputs described by (19). Linearization algorithm for MIMO system is an extension of SISO algorithm onto the case of multidimensional system and consists of following steps:

Step 1. From Definition 6 (as in one-dimensional case) we determine a relative degree of nonlinear system by calculating the Lie derivative from the function $h_{i}(\mathbf{x}),(1 \leq i \leq m)$ along the vector fields $\mathbf{f}$ and $\mathbf{g}_{i}(\mathbf{x})$, respectively.

Note 10. To determine the relative degree of nonlinear multidimensional system we differentiate $j^{\text {th }}$ outputs:

$$
\dot{y}_{j}=L_{\mathbf{f}} h_{j}(\mathbf{x})+\sum_{i=1}^{m}\left(L_{\mathbf{g}_{i}} h_{j}(\mathbf{x})\right) u_{i}
$$

If $L_{\mathbf{g}_{i}} h_{j}(\mathbf{x})=0$ then for each $i$ differentiation is carried out until in the ith step for some natural $r$ we obtain that $L_{\mathbf{g}_{i}} L_{\mathbf{f}}^{r_{j}-1} h_{j}(\mathbf{x}) \neq 0$.

To differentiate outputs we use general relation:

$$
y_{j}{ }^{(i)}=L_{\mathbf{f}}^{r_{j}} h_{j}(\mathbf{x})+\sum_{i=1}^{m} L_{\mathbf{g}} L_{\mathbf{f}}^{r_{j}-1} h_{j}(\mathbf{x}) u_{i}
$$

If $L_{\mathbf{g}_{i}} L_{\mathbf{f}}^{r_{j}-1} h_{j}(\mathbf{x}) \neq 0$, then the output equation of linearized system is written as

$$
\left[\begin{array}{c}
y_{j}^{r_{1}} \\
y_{j}^{r_{2}} \\
\vdots \\
y_{j}^{r_{m}}
\end{array}\right]=\left[\begin{array}{c}
L_{f}^{r_{1}} h_{1} \\
L_{f}^{r_{2}} h_{2} \\
\vdots \\
L_{f}^{r_{m}} h_{m}
\end{array}\right]+E(\mathbf{x})\left[\begin{array}{c}
u_{1} \\
u_{2} \\
\vdots \\
u_{m}
\end{array}\right]
$$

where $E(\mathbf{x})$ is $m \times m$ decoupling matrix defined by (11).

As a result we obtain the relative degree of the system $r=$ $r_{1}+\ldots+r_{p}$, for $1 \leq p \leq m$.

Step 2. We determine the decoupling matrix (11) and vector (16).

Step 3. If $\operatorname{det} \mathbf{E}(\mathbf{x}) \neq 0$, then matrix $\mathbf{E}(\mathbf{x})$ is nonsingular we determine the feedback linearizing the system as $\mathbf{u}=$ $-(\mathbf{E}(\mathbf{x}))^{-1} \circ \mathbf{b}(\mathbf{x})+(\mathbf{E}(\mathbf{x}))^{-1} \circ \mathbf{v}$

Step 4. From a general relation we determine the transformation of state variables $S(\mathbf{x})$ for a multidimensional system: $S_{k}^{i}(\mathbf{x})=L_{\mathbf{f}}^{k-1} h_{i}(\mathbf{x})$.

Step 5. We determine state variables using $z_{k}^{i}(\mathbf{x})=L_{\mathbf{f}}^{k-1} h_{i}(\mathbf{x})$.

Step 6. We determine the state equation of linearized system and the equations of system output.

Example 11. The functionality of the algorithm is presented by electrical circuit with two nonlinear elements, (Figure 3).

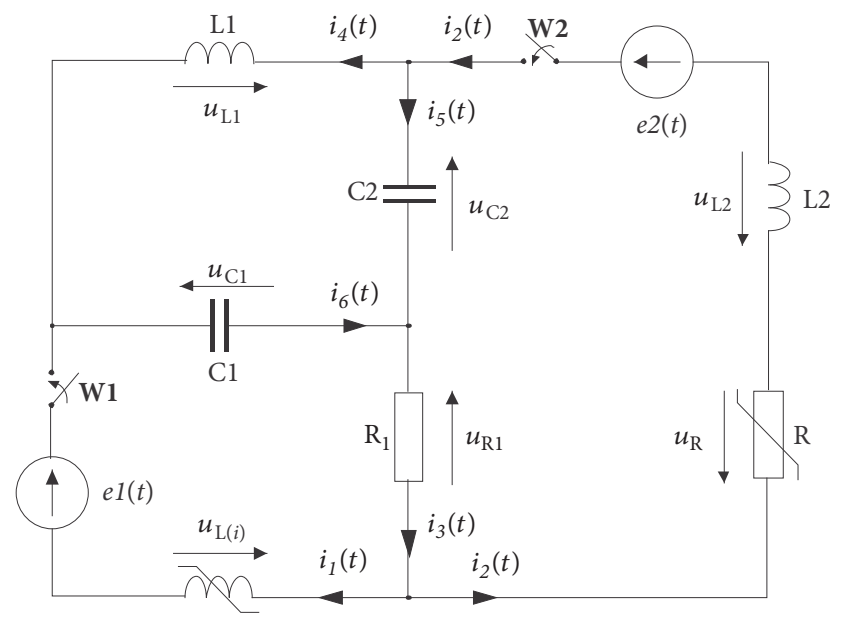

FIgURE 3: Diagram of nonlinear circuit of the 5th order with two power sources.

We assume that the initial state of the circuit is equal to zero and at $t=0$ we close switches $W_{1}$ i $W_{2}$. simultaneously. We also assume that current-voltage characteristics of nonlinear resistive element $u=b \cdot i \cdot \ln i$, where $b$ is a coefficient of $\left(\Omega A^{-1}\right)$ dimension. Nonlinear coil (without loss) is given by $u=L(i)(d i / d t)$, where $L(i)=(a \cdot i)^{-1}$ and $a$ is a coefficient of $\left(\Omega A^{-1}\right)$ dimension.

We assume that $i_{1}=x_{1}, i_{2}=x_{2}, i_{4}=x_{3}, u_{\mathrm{C} 1}=$ $x_{4}, u_{C 2}=x_{5}$ are state variables. We order variables and introduce notations:

$$
R_{1} / L_{2}=a ; R_{1} / L_{2}=b ; 1 / L_{1}=c ; 1 / C_{1}=w, 1 / C_{2}=
$$
$l, 1 / L_{2}=k$ and the expression for the inductance of nonlinear coil $L\left(i_{1}\right)=L\left(x_{1}\right)=\left(x_{1}\right)^{-1}$ to obtain the model system of equations in which the vectors $\mathbf{f}(\mathbf{x}), \mathbf{g}_{1}(\mathbf{x}), \mathbf{g}_{2}(\mathbf{x})$ and $(\mathbf{f}, \mathbf{g} \in$ $\left.\mathbf{R}^{5}, n=5\right)$ become

$$
\mathbf{f}(\mathbf{x})=\left[\begin{array}{c}
-x_{1} x_{4}-R_{1} \cdot\left(x_{1}\right)^{2}+R_{1} x_{1} x_{2} \\
-a\left(x_{1}+x_{2}\right)-b \cdot \ln x_{2}+k x_{5} \\
-c \cdot x_{4}+c \cdot x_{5} \\
w \cdot x_{1}+w \cdot x_{3} \\
l \cdot x_{2}-l \cdot x_{3}
\end{array}\right] ;
$$

$\mathbf{g}_{1}(\mathbf{x})=\left[\begin{array}{c}x_{1} \\ 0 \\ 0 \\ 0 \\ 0\end{array}\right]$ 
The output equations are

$$
\begin{aligned}
& y_{1}=x_{3}, \\
& y_{2}=x_{5} .
\end{aligned}
$$

The considered system has two outputs and two inputs. The first step of the linearization algorithm is to determine a relative degree of the system. For function $h_{1}(\mathbf{x})$ we obtain

$$
\begin{aligned}
& L_{\mathbf{g}_{1}} h_{1}(\mathbf{x})=\left[\begin{array}{lllll}
0 & 0 & 1 & 0 & 0
\end{array}\right]\left[\begin{array}{lllll}
x_{1} & 0 & 0 & 0 & 0
\end{array}\right]^{\mathrm{T}}=0 \\
& L_{\mathbf{g}_{2}} h_{1}(\mathbf{x})=\left[\begin{array}{lllll}
0 & 0 & 1 & 0 & 0
\end{array}\right]\left[\begin{array}{lllll}
0 & k & 0 & 0 & 0
\end{array}\right]^{\mathrm{T}}=0
\end{aligned}
$$

Similarly, for the function $h_{2}(\mathbf{x})$ we get

$$
\begin{aligned}
& L_{\mathbf{g}_{1}} h_{2}(\mathbf{x})=\left[\begin{array}{lllll}
0 & 0 & 0 & 0 & 1
\end{array}\right]\left[\begin{array}{lllll}
x_{1} & 0 & 0 & 0 & 0
\end{array}\right]^{\mathrm{T}}=0 \\
& L_{\mathbf{g}_{2}} h_{2}(\mathbf{x})=\left[\begin{array}{lllll}
0 & 0 & 0 & 0 & 1
\end{array}\right]\left[\begin{array}{lllll}
0 & k & 0 & 0 & 0
\end{array}\right]^{\mathrm{T}}=0
\end{aligned}
$$

As the first derivatives are equal to zero, we calculate derivatives of consecutive rows. For the function $h_{1}(\mathbf{x})$ we get

$$
\begin{aligned}
& L_{g_{1}} L_{\mathbf{f}} h_{1}(\mathbf{x})=L_{g_{1}}\left(-c x_{4}+c x_{5}\right)=0 \\
& L_{g_{2}} L_{\mathbf{f}} h_{1}(\mathbf{x})=L_{g_{2}}\left(-c x_{4}+c x_{5}\right)=0 \\
& L_{g_{1}} L_{\mathbf{f}}{ }^{2} h_{1}(\mathbf{x}) \\
& \quad=\left[\begin{array}{llll}
-c w & l c-c w-l c & 0 & 0
\end{array}\right]\left[\begin{array}{lllll}
x_{1} & 0 & 0 & 0 & 0
\end{array}\right]^{\mathrm{T}} \\
& \quad=-c \cdot w \cdot x_{1} \neq 0 \\
& L_{g_{2}} L_{\mathbf{f}}{ }^{2} h_{1}(\mathbf{x}) \\
& \quad=\left[\begin{array}{lllll}
-c w & l c-c w-l c & 0 & 0
\end{array}\right]\left[\begin{array}{lllll}
0 & k & 0 & 0 & 0
\end{array}\right]^{\mathrm{T}} \\
& \quad=k \cdot l \cdot c \neq 0
\end{aligned}
$$

As a result the relative degree of subsystem $r_{1}=3$. From the calculations for the function $h_{2}(\mathbf{x})$ we obtain $r_{2}=2$ because

$$
\begin{aligned}
& L_{g_{1}} L_{\mathbf{f}} h_{2}(\mathbf{x})=L_{g_{1}}\left(l \cdot x_{2}-l \cdot x_{3}\right)=0 \\
& L_{g_{2}} L_{\mathbf{f}} h_{2}(\mathbf{x})=L_{g_{2}}\left(l \cdot x_{2}-l \cdot x_{3}\right)
\end{aligned}
$$

$$
=\left[\begin{array}{lllll}
0 & l & -l & 0 & 0
\end{array}\right]\left[\begin{array}{lllll}
0 & k & 0 & 0 & 0
\end{array}\right]^{\mathrm{T}}=k \cdot l
$$$$
\neq 0
$$

Finally, we find that the relative (vector) degree of the considered system equals $\left\{r_{1}, r_{2}\right\}=\{3,2\}$. As $r_{1}+r_{2}=n$ the next step of the algorithm is to determine decoupling (decomposing) matrix $\mathbf{E}(\mathbf{x})$ :

$$
\mathbf{E}(\mathbf{x})=\left[\begin{array}{cc}
c \cdot w \cdot x_{1} & -k \cdot l \cdot c \\
0 & k \cdot l
\end{array}\right]
$$

Since the determinant of the matrix $\operatorname{det} \mathbf{E}(\mathbf{x}) \neq 0, \mathbf{E}(\mathbf{x})$ is nonsingular and we can determine feedback linearizing output-input system:

$$
\left[\begin{array}{l}
e_{1} \\
e_{2}
\end{array}\right]=\mathbf{E}^{-1}(\mathbf{x})\left[\left(\begin{array}{l}
v_{1} \\
v_{2}
\end{array}\right)-\left(\begin{array}{l}
b_{1}(\mathbf{x}) \\
b_{2}(\mathbf{x})
\end{array}\right)\right]=\frac{1}{k \cdot l \cdot c \cdot w \cdot x_{1}}
$$$$
\cdot\left[\begin{array}{cc}
k \cdot l & k \cdot l \cdot c \\
0 & c \cdot w \cdot x_{1}
\end{array}\right]\left[\left(\begin{array}{c}
v_{1} \\
v_{2}
\end{array}\right)-\left(\begin{array}{c}
L_{\mathbf{f}}^{3} h_{1}(\mathbf{x}) \\
L_{\mathbf{f}}^{2} h_{2}(\mathbf{x})
\end{array}\right)\right]
$$

where $v_{1} \mathrm{i} v_{2}$ are components of a new supply (output) vector.

After transformations we get

$$
\begin{array}{r}
v_{1}=b_{1}(\mathbf{x})+a_{11}(\mathbf{x}) \cdot e_{1}+a_{12}(\mathbf{x}) \cdot e_{2} \\
\text { and } v_{2}=b_{2}(\mathbf{x})+a_{21}(\mathbf{x}) \cdot e_{1}+a_{22}(\mathbf{x}) \cdot e_{2}
\end{array}
$$

New coordinates are given by

$$
\begin{aligned}
z_{1}(\mathbf{x}) & =S_{1}^{1}(\mathbf{x})=h_{1}(\mathbf{x})=x_{3} \\
z_{2}(\mathbf{x}) & =S_{2}^{1}(\mathbf{x})=L_{\mathbf{f}} h_{1}(\mathbf{x})=-c \cdot x_{4}+c \cdot x_{5} \\
z_{3}(\mathbf{x}) & =S_{3}^{1}(\mathbf{x})=L_{\mathbf{f}}{ }^{2} h_{1}(\mathbf{x}) \\
& =c \cdot w \cdot x_{1}+l \cdot c \cdot x_{2}-x_{3}(l \cdot c+w \cdot c) \\
z_{4}(\mathbf{x}) & =S_{1}^{2}(\mathbf{x})=h_{2}(\mathbf{x})=x_{5} \\
z_{5}(\mathbf{x}) & =S_{2}^{2}(\mathbf{x})=L_{\mathbf{f}} h_{2}(\mathbf{x})=l \cdot x_{2}-l \cdot x_{3}
\end{aligned}
$$


The normal form of linearized system according to (14) is

$$
\begin{aligned}
\dot{z}_{1}(\mathbf{x})= & \frac{d}{d t} h_{1}(\mathbf{x})=\frac{d}{d t}\left(x_{3}\right)=\dot{x}_{3}=-c \cdot x_{4}+c \cdot x_{5} \\
= & z_{2}(\mathbf{x}) \\
\dot{z}_{2}(\mathbf{x})= & \frac{d}{d t}\left(L_{\mathbf{f}} h_{1}(\mathbf{x})\right)=\frac{d}{d t}\left(-c \cdot x_{4}+c \cdot x_{5}\right) \\
= & -c \cdot \dot{x}_{4}+c \cdot \dot{x}_{5} \\
= & -c\left(w x_{1}+w x_{3}\right)+c\left(l x_{2}+l x_{3}\right)=z_{3}(\mathbf{x}) \\
\dot{z}_{3}(\mathbf{x})= & \frac{d}{d t}\left(L_{\mathbf{f}}^{2} h_{1}(\mathbf{x})\right) \\
= & c \cdot w \cdot \dot{x}_{1}+l \cdot c \cdot \dot{x}_{2}-\dot{x}_{3}(l \cdot c+w \cdot c) \\
= & c w\left(-x_{1} x_{4}-R_{1} \cdot\left(x_{1}\right)^{2}+R_{1} x_{1} x_{2}+x_{1} e_{1}\right) \\
& +l c\left(-a\left(x_{1}+x_{2}\right)-b \cdot \ln x_{2}+k x_{5}+k e_{2}\right) \\
& +\left(c \cdot x_{4}-c \cdot x_{5}\right)(l \cdot c+w \cdot c)=v_{1} \\
\dot{z}_{5}(\mathbf{x})= & \frac{d}{d t}\left(L_{\mathbf{f}} h_{2}(\mathbf{x})\right)=\frac{d}{d t}\left(l \cdot x_{2}-l \cdot x_{3}\right) \\
= & l \cdot \dot{x}_{2}-l \cdot \dot{x}_{3} \\
= & l\left(-a\left(x_{1}+x_{2}\right)-b \cdot \ln x_{2}+k x_{5}\right)+k \cdot e_{2}-l \\
\dot{z}_{4}(\mathbf{x})= & \frac{d}{d t} h_{2}(\mathbf{x})=\frac{d}{d t}\left(x_{5}\right)=\dot{x}_{5}=l \cdot x_{2}-l \cdot x_{3} \\
= & z_{5}(\mathbf{x}) \\
& \\
&
\end{aligned}
$$

Also, the structure of the assumed power supply implies that $y_{1}=z_{1}$ and $y_{2}=z_{3}$

Finally, we obtain the linear form of the state equations for the system in which the ith input $y_{i}$ depends only on the ith power input $v_{i}$, for $i=1,2$ is obtained.

\section{Conclusion}

The presented examples show that the equivalent linear models are obtained by applying the developed transformation algorithms with feedback linearization to mathematical models. Thus, the linear models can be analysed using methods known from the theory of linear systems. The obtained results can be applied to a nonlinear system (1) using the inverse transformation $S^{-1}(\mathbf{z})$. This methodology can be used to precisely analyse the spark discharge as a dynamic phenomenon in the ignition system of a motor vehicle, which allows for high-precision response [54].

It is worth noting that proper fulfillment of the Theorem 3 determines how the transformation directly linearizing the system, or the transformation with feedback should be sought.

It should be stressed that the numbers $r_{1}+\ldots+r_{p}$ are called the characteristic numbers and any characteristic number $r_{i}$ is associated with $\mathrm{j}$-th output of the system $h_{i}$ which determines how many times $y_{i}$ must be differentiated to get the power $u_{i}$ explicite.

Nonlinear system with multiple inputs and multiple outputs can be decomposed by a static feedback only if the matrix $\mathbf{E}(\mathbf{x})$ is nonsingular.

Therefore, nonsingularity of this matrix is a prerequisite for a solution of the problem. However, when choosing different output functions of the test system, to find a regular feedback, it often turns out that the decoupling matrix is singular.

\section{Data Availability}

The data used to support the findings of this study are available from the corresponding author upon request.

\section{Conflicts of Interest}

The authors declare that they have no conflicts of interest.

\section{References}

[1] A. Isidori, Nonlinear Control Systems: An Introduction, Springer, Berlin, Germany, 1989.

[2] A. Isidori, Nonlinear Control Systems, Springer, Berlin, Germany, 1995.

[3] H. Nijmeijer and A. van der Schaft, Nonlinear Dynamical Control Systems, Springer, NY, USA, Verlag, Germany, 1990.

[4] L. R. Hunt, R. J. Su, and G. Meyer, "Global transformations of nonlinear systems," IEEE Transactions on Automatic Control, vol. 28, no. 1, pp. 24-31, 1983.

[5] N. Bourbaki, Lie groups and Lie algebras, Springer, Berlin, Germany, 1989, Chapters 1-3.

[6] N. Bourbaki, Lie groups and Lie algebras, Springer, Berlin, Germany, 2002, Chapters 4-6.

[7] K. Fujimoto and T. Sugie, "Freedom in coordinate transformation for exact linearization and its application to transient behavior improvement," Automatica, vol. 37, no. 1, pp. 137-144, 2001.

[8] M. Guay, "Observer linearization of nonlinear systems by generalized tansformations," Asian Journal of Control, vol. 7, no. 2, pp. 187-196, 2005.

[9] A. J. Jordan and J. P. Nowacki, "Global linearization of nonlinear state equations," International Journal of Applied Electromagnetics and Mechanics, vol. 19, no. 1-4, pp. 637-642, 2004.

[10] M. Bodson and J. Chiasson, "Differential-geometric methods for control of electric motors," International Journal of Robust and Nonlinear Control, vol. 8, no. 11, pp. 923-954, 1998.

[11] A. Zawadzki, "Application of local coordinates rectification in linearization of selected parameters of dynamic nonlinear systems," COMPEL. The International Journal for Computation and Mathematics in Electrical and Electronic Engineering, vol. 33, no. 5, pp. 1819-1830, 2014.

[12] A. Zawadzki and S. Różowicz, "Application of input - State of the system transformation for linearization of some nonlinear generators," International Journal of Control, Automation and Systems, vol. 13, no. 3, pp. 626-633, 2015.

[13] A. Zawadzki, "Comparative analysis of methods for determining the transformation linearizing the nonlinear equations of 
the system state," Przegląd Elektrotechniczny, vol. 4, pp. 28-33, 2014.

[14] B. Jakubczyk and W. Respondek, "On linearization of control systems," Bulletin de l'Académie Polonaise, Série des Sciences Mathématiques, vol. 28, pp. 517-522, 1980.

[15] P. Yat-Sun and P. Wai-Yin, "Application of Elementary," Differential Geometry to Influence Analysis, 2012.

[16] S. Čelikovský and H. Nijmeijer, "Equivalence of nonlinear systems to triangular form: the singular case," Systems \& Control Letters, vol. 27, no. 3, pp. 135-144, 1996.

[17] A. J. Krener, "Approximate linearization by state feedback and coordinate change," Systems \& Control Letters, vol. 5, no. 3, pp. 181-185, 1984.

[18] A. De Luca, "Decoupling and feedback linearization of robots with mixed rigid/elastic joints," International Journal of Robust and Nonlinear Control, vol. 8, no. 11, pp. 965-977, 1998.

[19] A. Isidori, C. Gori-Giorgi, and S. Monaco, "Nonlinear Decoupling via Feedback: A Differential Geometric Approach," IEEE Transactions on Automatic Control, vol. 26, no. 2, pp. 331-345, 1981.

[20] I. A. Tall and W. Respondek, "Feedback Linearizable Strict Feedforward Systems," in Proceedings of the 47th IEEE Conference on Decision and Control, pp. 2499-2504, Mexico, 2008.

[21] T. K. Boukas and T. G. Habetler, "High-performance induction motor speed control using exact feedback linearization with state and state derivative feedback," IEEE Transactions on Power Electronics, vol. 19, no. 4, pp. 1022-1028, 2004.

[22] B. Fangt and A. G. Kelka, "Exact Linearization of Nonlinear Systems by Time Scale," in IEEE Transformation Proceedings of the American Control Conference, pp. 3555-3560, Denver, Colorado, USA, 2003.

[23] K.-P. Liu, W. You, and Y.-C. Li, "Combining a feedback linearization approach with input shaping for flexible manipulator control," in Proceedings of the International Conference on Machine Learning and Cybernetics, pp. 561-565, China, 2003.

[24] D. Wang and M. Vidyasagar, "Control of a class of manipulators with the last link flexible - Part I: Feedback linearization," ASME Journal of Dynamic Systems, Measurement, and Control, vol. 113, no. 4 , pp. 655-661, 1991.

[25] J. Deutscher and C. Schmid, "A state space embedding approach to approximate feedback linearization of single input nonlinear control systems," International Journal of Robust and Nonlinear Control, vol. 16, no. 9, pp. 421-440, 2006.

[26] R. Devanathan, "Linearization condition through state feedback," Institute of Electrical and Electronics Engineers Transactions on Automatic Control, vol. 46, no. 8, pp. 1257-1260, 2001.

[27] A. Zawadzki and S. Różowicz, "Application of Input-State of the System Transformation for Linearization of Selected Electrical Circuits," Journal of Electrical Engineering, vol. 67, no. 3, pp. 199-205, 2016.

[28] B. Bonnard, "Quadratic control systems," Mathematics of Control, Signals, and Systems, vol. 4, no. 2, pp. 139-1606, 1991.

[29] B. Jakubczyk, "Equivalence and invariants of nonlinear control systems," in Nonlinear Controllability and Optimal Control, H. J. Sussmann, Ed., vol. 133, pp. 177-218, Marcel Dekker, NY, USA, Basel, Switzerland, 1990.

[30] B. Jakubczyk and W. Respondek, "Feedback classification of analytic control systems in the plane," Analysis of Controlled Dynamical Systems, pp. 263-273, 1991.

[31] B. Bonnard, J.-B. Caillau, and R. Dujol, "Energy minimization of single input orbit transfer by averaging and continuation,"
Bulletin des Sciences Mathématiques, vol. 130, no. 8, pp. 707-719, 2006.

[32] R. B. Gardner, "The Method of Equivalence and Its Applications," in Proceedings of the CBMS Regional Conference Series in Applied Mathematics, vol. 58, SIAM, Philadelphia, USA, 1989.

[33] R. B. Gardner, W. F. Shadwick, and G. R. Wilkens, "Geometric isomorphism with applications to closed loop controls," SIAM Journal on Control and Optimization, vol. 27, no. 6, pp. 1361$1368,1989$.

[34] R. B. Gardner and W. F. Shadwick, "The GS algorithm for exact linearization to Brunovsky normal form," IEEE Transactions on Automatic Control, vol. 37, no. 2, pp. 224-230, 1992.

[35] R. B. Gardner, W. F. Shadwick, and G. R. Wilkens, "Feedback equivalence and symmetries of Brunovsky normal forms," Systems \& Control Letters, vol. 97, pp. 115-130, 1989.

[36] I. Kupka, "On feedback equivalence," in Proceedings of the Differential Geometry, Global Analysis, and Topology CMS Conference, vol. 12, pp. 105-117, AMS, Providence, RI, USA, 1991.

[37] B. Bonnard, "Feedback equivalence for nonlinear systems and the time optimal control problem," SIAM Journal on Control and Optimization, vol. 29, no. 6, pp. 1300-1321, 1991.

[38] B. Bonnard, "Quadratic control systems," Mathematics of Control, Signals, and Systems, vol. 4, no. 2, pp. 139-160, 1991.

[39] B. Jakubczyk, "Feedback invariants, critical trajectories, and Hamiltonian formalism," in Nonlinear Control in the Year 2000, vol. 258 of Lecture Notes in Control and Inform. Sci., pp. 545568, Springer-Verlag, London, UK, 2001.

[40] B. Jakubczyk, "Critical Hamiltonians and feedback invariants," in Geometry of Feedback and Optimal Control, B. Jakubczyk and W. Respondek, Eds., pp. 219-256, Marcel Dekker, NY, USA, Basel, Switzerland, 1998.

[41] A. Banaszuk and J. Hauser, "Approximate feedback linearization: homotopy operator approach," in Proceedings of the American Control Conference, pp. 1690-1694, Baltimore, Maryland, USA, 1994.

[42] G. O. Guardabassi and S. M. Savaresi, "Approximate linearization via feedback-an overview," Automatica, vol. 37, no. 1, pp. $1-15,2001$.

[43] K. Guemghar, B. Srinivasan, and D. Bonvin, "Approximate input-output linearization of nonlinear systems using the observability normal form," in Proceedings of the European Conference Control, 2003.

[44] R. W. Brockett, "Feedback invariants for nonlinear systems," in Proceedings of the 6th IFAC World Congr., vol. 6, pp. 1115-1120, Helsinki, Finland, 1978.

[45] W. Kang, "Approximate linearization of nonlinear control systems," Systems \& Control Letters, vol. 23, no. 3, pp. 43-52, 1994.

[46] W. Kang and A. J. Krener, "Extended quadratic controller normal form and dynamic state feedback linearization of nonlinear systems," SIAM Journal on Control and Optimization, vol. 30, no. 6, pp. 1319-1337, 1992.

[47] D. A. Lawrence, "Input-Output Pseudolinearization for Nonlinear Systems," IEEE Transactions on Automatic Control, vol. 39, no. 11, pp. 2207-2218, 1994.

[48] W. Respondek and I. A. Tall, "Nonlinearizable single-input control systems do not admit stationary symmetries," Systems \& Control Letters, vol. 46, no. 1, pp. 1-16, 2002.

[49] I. A. Tall, "Multi-Input Control Systems: Explicit Feedback Linearization," in Proceedings of the 49th IEEE Conference on Decision and Control, Hilton Atlanta Hotel, Atlanta, GA, USA, 2010. 
[50] I. A. Tall, "State and feedback linearizations of single-input control systems," Systems \& Control Letters, vol. 59, no. 7, pp. 429-441, 2010.

[51] D. Bump, Lie Groups, Graduate Texts in Mathematics, vol. 225, Springer, NY, USA, 2004.

[52] J.-P. Serre, Complex semisimple Lie algebras, Springer-Verlag, Berlin, Germany, 2001.

[53] D. Cheng, A. Isidori, W. Respondek, and T. J. Tarn, "Exact linearization of nonlinear systems with outputs," Mathematical Systems Theory, vol. 21, no. 1, pp. 63-83, 1988.

[54] S. Rózowicz and S. Tofil, "The influence of impurities on the operation of selected fuel ignition systems in combustion engines," Archives of Electrical Engineering, vol. 65, no. 2, pp. 349-360, 2016. 


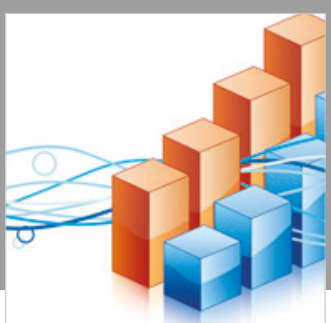

Advances in

Operations Research

\section{-n-m}
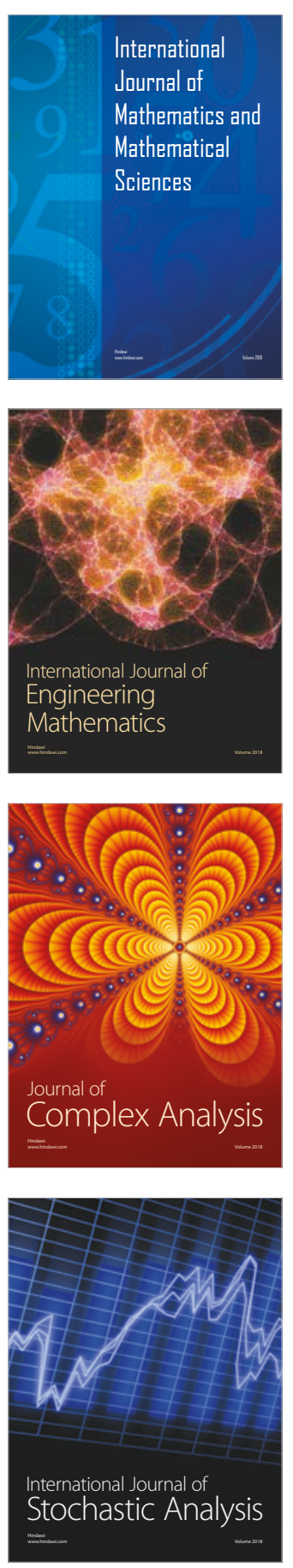
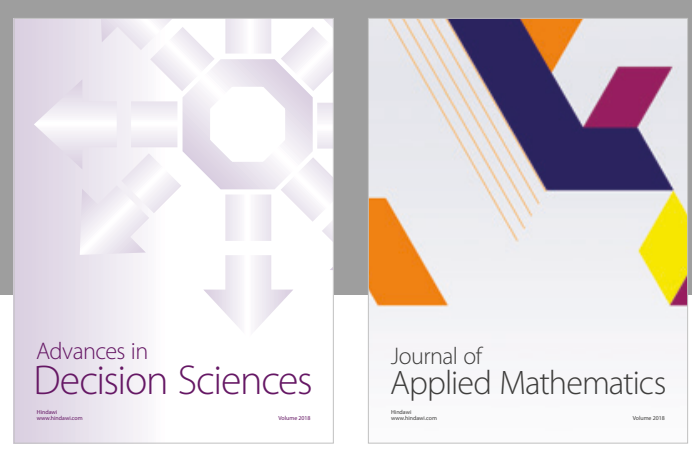

Journal of

Applied Mathematics
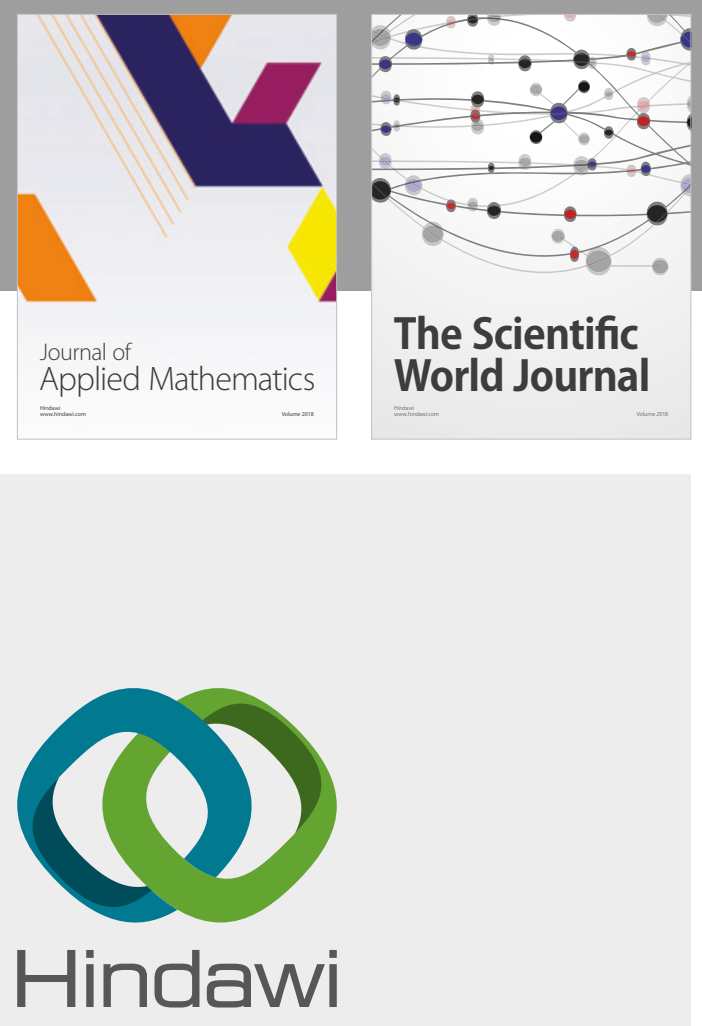

Submit your manuscripts at

www.hindawi.com

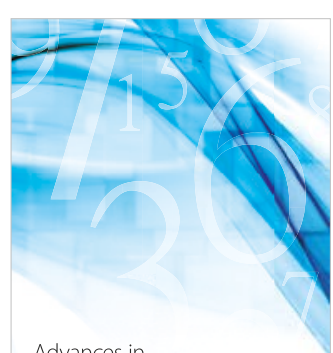

Advances in
Numerical Analysis
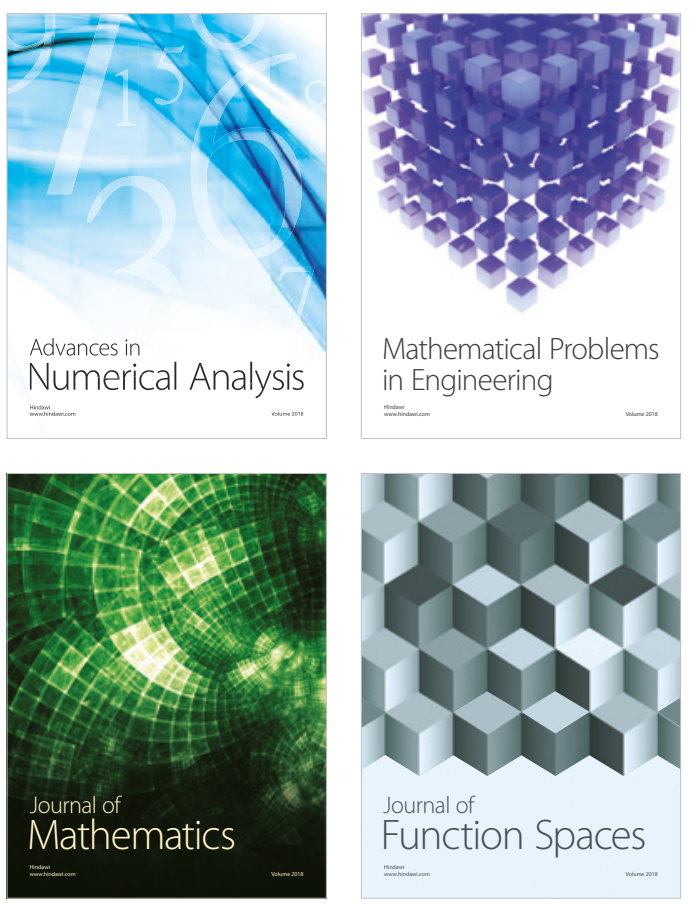

Mathematical Problems in Engineering

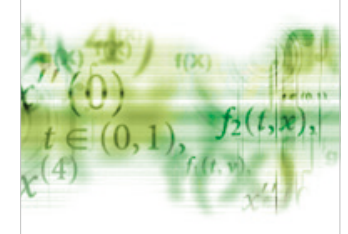

International Journal of

Differential Equations

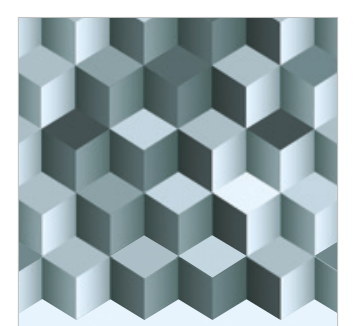

Journal of

Function Spaces

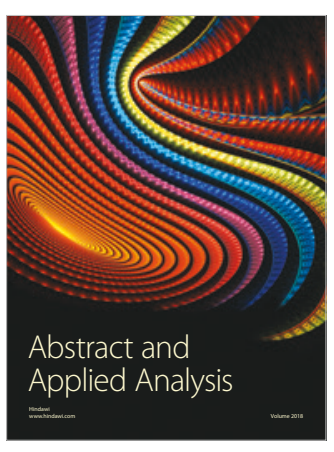

The Scientific

World Journal

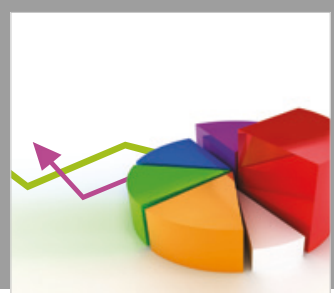

Journal of

Probability and Statistics
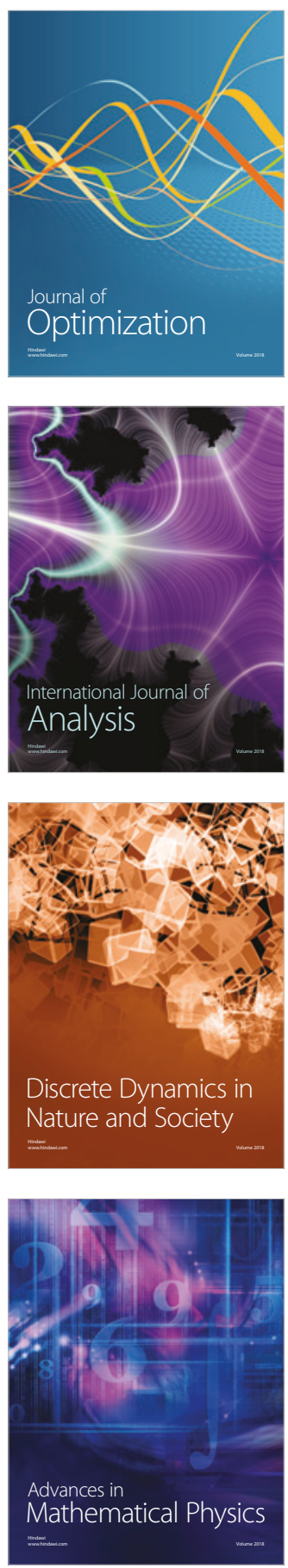